\title{
Ueber das normale Vorkommen des Jods im Thierkörper.
}

(II. Mittheilung.)

Von

E. Baumann und E. Roos.

\section{Darstellung des Thyrojodins.}

Bei unseren Versuchen, die wirksame Substanz der Schilddrüse zu isoliren, benützten wir als Erkennungsmerkmal, ob jeweils dieser Stoff in den von uns gewonnenen Producten enthalten sei, seine Wirksamkeit auf parenchymatöse Kröpfe, welche hier in genügender Zahl zur Verfügung stehen. Diese von Emminghaus und Reinhold zuerst beobachtete Reaction ist eine so prompte, dass wir in der Regel schon nach 2-4 Tagen im Stande waren, über den Grad der Wirksamkeit der untersuchten Präparate uns eine Vorstellung zu bilden.

Dit Mehrzahl dieser Beobachtungen ist an Patienten der Poliklinik und bei Insassen der hiesigen Kreispflegeanstalt gemacht worden. Wir sind den Directoren dieser Anstalten, Herrn Prof. Dr. Thom as und Herrn Medicinalrath Dr. Es ch bacher, für die bereitwillige Förderung, welche sie unseren Versuchen gewährten, zu lebhaftem Danke verpflichtet.

Die von uns geprüften Präparate sind zum grossen Theil in den Farbenfabriken vorm. Friedr. Bayer \& Go. in Elberfeld nach unseren Angaben dargestellt worden, wofür wir denselben um so dankbarer sind, als die hier zu Gebote stehenden Mengen von Hammelschilddrüsen bei Weitem nicht hinreichten.

Nachdem der wirksame Bestandtheil der Schilddrüsen als eine organische Jodverbindung erkannt war, welcher «Thyrojodin» benannt wurde'), war eine zweite Reaction gegeben, mit deren Hilfe noch bequemer als mit der ersteren die Isolirung desselben verfolgt werden konnte.

1) Ba umann, diese Zeitschrift, Bd. 21, S. 319. 
Bei allen Versuchen gingen wir von der grundlegenden Beobachtung aus, dass der wirksame Stoff der Schilddrüse weder durch Erhitzen auf $100^{\circ}$, noch durch die Einwirkung starker Mineralsäuren zerstört wird. Werden zerkleinerte Schilddrüsen mit 10 procentiger Salzsäure übergossen und einige Zeit damit in Berührung gelassen, so ist die wirksame Substanz noch so gut wie ungemindert vorhanden. Als nämlich die Salzsäure mit Natronlauge neutralisirt und eingedampft wurde, zeigte der trockene Rückstand in Quantitäten, welche einem Gramm der frischen Drüsen entsprachen, noch eine sehr prägnante Wirkung auf Kröpfe.

Beim Kochen der Schilddrüsen mit verd. Schwefelsäure (1:10) erfolgt nach einigen Stunden nahezu völlige Lösung, so zwar, dass in der Flüssigkeit ein feinflockiger Niederschlag suspendirt ist, dessen Menge bei längerem Kochen sich langsam noch vermindert. Das immer im Innern der Drüse noch vorhandene Fett ${ }^{1}$ ) schwimmt zum grössten Theil an der Oberfläche und kann nach, dem Erkalten durch Coliren durch feinmaschige Gaze mechanisch abgetrennt werden. Wir verwendeten von der Schwefclsäure etwa das 4 fache von dem Gewichte der frischen Drüsen. Auch hierbei zeigte sich, dass die wirksame Substanz selbst nach 3 tägigem Kochen nicht zerstört wurde. Sie ging aber fast nicht in die Lösung über, sondern fand sich zum grössten Theil in dem bräunlichen Niederschlag, welcher nach dem Erkalten von der sauren Flüssigkeit abfiltrirt wurde. Die Menge dieses Niederschlages beträgt je nach der Dauer des Kochens ca. $\%$ bis $1 \%$, Procent vom Gewichte der verarbeiteten Drüsen.

Die von dem Ungelösten abfiltrirte saure Flüssigkeit, welche braun gefärbt ist, enthält einen kleineren Theil des Thyrojodins gelöst. Wird durch Neutralisiren mit Natronlauge die freie Säure bis auf einen kleinen Rest abgestumpft, so scheidet sich beim Eindampfen auf $1 / 5$ bis $1 / 6$ des Volums der Flüssigkeit nach dem Erkalten der grössere Theil des gelösten Thyrojodins ab. Diese Abscheidung ist noch vollkommener,

1) Vor der Verarbeitung wurden die Drüsen jeweils sorgfältig präparirt. 
wenn man den grössten Theil der Schwefelsäure mit Baryumcarbonat entfernt und das Filtrat einengt.

Die von diesem Niederschlag abfiltrirte Flüssigkeit enthält noch immer etwas Tyrojodin, und wenn sie in grösseren Mengen eingegeben wird, lässt sich eine wenn auch sehr schwache Wirksamkeit auf Kröpfe noch erkennen.

Die letzten Antheile der Jodverbindung werden der eingedampften Mutterlauge durch Auskochen mit Alkohol, welcher das Thyrojodin aufnimmt, entzogen, wobei allerdings auch noch andere Stoffe in Lösung gehen, deren völlige Abscheidung nicht leicht gelingt.

Die Hauptmenge der wirksamen Substanz ist in dem bei der Einwirkung der Schwefelsäure auf die Drüsen ungelöst gebliebenen Rückstande enthalten. Bevor man ihn abfiltrirt, wird die Flüssigkeit einige Stunden in Eiswasser abgekühlt.

Dieses Product enthält aber noch Fette, Fettsäuren und andere Stoffe neben dem Thyrojodin. Zur weiteren Reinigung der letzteren wird der noch feuchte Niederschlag mit viel Alkohol von $90 \%$ wiederholt ausgekocht, wobei das Thyrojodin allmälig völlig in Lösung geht. Die weingeistigen Extracte werden im Wasserbade eingedunstet, mit dem ca. 10 fachen Gewicht von Milchzucker verrieben und zur Entfernung der Fette und Fettsäuren mit Petroläther oder mit einem Gemenge von wasserfreiem Aether und Petroläther ausgezogen. Die Verreibung mit Milchzucker erleichtert hierbei sehr wesentlich die Entfernung der fettartigen Substanzen.

Die Abscheidung des Thyrojodins von dem Milchzucker geschieht in der Art, dass die Verreibung in wenig kalter verdünnter Natronlauge gelöst (und wenn nöthig filtrirt) wird; beim Ansäuern der alkalischen Flüssigkeit wird das Thyrojodin in Flocken abgeschieden. Es wird abfiltrirt, ausgewaschen, wieder mit Natronlauge gelöst, und mit Salzsäure oder Schwefelsäure aufs Neue gefällt. Durch diese Behandlung wird ein Theil der beigemengten gefärbten Stoffe - bei nur geringem Verlust an Thyrojodin - beseiligt.

Das soweit gereinigte Thyrojodin stellt nach dem Trocknen ein bräunlich gefärbtes Pulver dar, welches in Wasser un- 
löslich ist, in heissem Weingeist schwer, in ätzenden Alkalien leicht sich auflöst. Es enthält reichlich Stickstoff, eine kleine Menge von Phosphor (ca. 0,5 Procent) und etwa 10 Procent Jod. Beim Erhitzen entwickelt es den Geruch von Pyridinbasen. Das Jod ist sehr fest gebunden. Durch Alkalien wird es nur schwer abgespalten. Auch Natriumamalgam bewirkt erst sehr allmälig die Abtrennung des Jods. Ueber die chemische Natur des Thyrojodins wird demnächst eingehender berichtet werden.

Dosen von 1 Milligramm des in der beschriebenen Art dargestellten Thyrojodins, welche einen Jodgehalt von je $1 / 10$ Milligramm besassen, zeigten nach 3 bis 4 maliger Application (in Milchzuckerverreibung) eine sehr deutliche und erhebliche Einwirkung auf Kröpfe. Diese Erfahrung beweist, wie schon früher betont wurde, dass die Thyrojodinwirkung nicht durch die geringen Mengen von Jod bedingt ist, welche in dem Thyrojodin dem Körper zugeführt werden, sondern durch die eigenartige organische Jodverbindung, welche unter normalen Verhältnissen in der Schilddrüse aus Spuren von Jodverbindungen, welche in der Nahrung enthalten sind, entsteht.

Bei der im Obigen geschilderten Art der Darstellung des Thyrojodins sind Verluste, welche 25-30 Procent von der Gesammtmenge der ursprünglich vorhandenen Jodverbindung betragen können, nicht ausgeschlossen. Dieser Verlust ist sehr viel geringer, wenn man die Abscheidung des Thyrojodins aus den Schilddrüsen durch die Verdauung mit künstlichem Magensaft bewirkt. Dabei erhält man auch ein weniger gefärbtes Product als beim Kochen mit Säuren.

Am Besten verwendet man künstlichen Magensaft (von 3 Promille $\mathrm{HCl}$ ) und lässt ihn 2 Tage lang bei $40^{\circ}$ auf die Schilddrüsen einwirken, deren Substanz dabei so gut wie ganz in Lösung geht, während das Thyrojodin fast völlig ungelöst bleibt, das dann, wie oben beschrieben, weiter gereinigt wird. Die bei der Verdauung gebildete Lösung von Hemialbumosen und Peptonen ist fast frei von Jod. Wird sie eingedunstet und bei Kröpfen geprüft, so zeigt sich eine ganz schwache Wirkung erst dann, wenn relativ grosse Quantitäten derselben verabreicht werden. 
Nachdem das Thyrojodin als der wirksame Bestandtheil der Schilddrüse erkannt war, erhob sich die weitere Frage, in welcher Form und Verbindung dasselbe in der Drüse selbst enthalten ist.

Dass es nur zum kleinsten Theil als solches, d. h. frei in der Drüse vorkommt, lehrt die Erfahrung, dass die auf verschiedenen Wegen dargestellten Wasser- oder GlycerinExtracte der Schilddrüse als wirksam erprobt worden sind, wobei freilich $\mathrm{zu}$ bemerken ist, dass die Intensität der Wirkung der bisher dargestellten Extracte stets schwächer ist als diejenige der äquivalenten Menge der frischen Drüsen.

Folgende Versuche geben über diese Verhältnisse näheren Aufschluss.

25 Gramm der frischen Hammelschilddrüsen werden gut zerkleinert und mit Alkohol 3 Mal ausgekocht. Der Rückstand der Alkohollösungen erwies sich als jodhaltig und die jodhaltige Verbindung als identisch mit dem Thyrojodin. Allein ihre Menge betrug nicht mehr, als etwa dem 15. Theil der Gesammtmenge des vorhandenen Jods entsprach. Je reicher die Schilddrüsen an Jod sind, um so grösser ist auch der Antheil des Thyrojodins, welches in der Drüse frei enthalten ist und durch Extraction mit Alkohol ihr entzogen werden kann.

Weitere Versuche lehrten bald, dass man den grösseren Theil der jodhaltigen Verbindung der Schilddrüse durch Extraction mit kaltem Wasser entziehen kann. Allein ein beträchtlicher Theil derselben bleibt in dem in Wasser unlöslichen Rückstande, welcher dem entsprechend schwächer als die frischen Drüsen, aber immer noch sehr deutlich, auch bei kleineren Dosen, auf Kröpfe einwirkte.

Etwas besser, aber keineswegs vollständig, wird aus der Schilddrüse die Jodverbindung aufgenommen, wenn man dieselbe mit Glycerin (gl. Theile Wasser und Glycerin) kalt extrahirt; auch hierbei enthält der unlösliche Rückstand - auch nach 3-4maliger Extraction - noch recht merkliche Mengen von Jod. Die Glycerinextracte liefern auf Zusatz von viel absolutem Alkohol eine Fällung, in welcher der grössere Theil der vorhandenen Jodverbindung enthalten ist. 
Weitere Versuche zeigten, dass das Thyrojodin in der Schilddrüse zum weitaus grössten Theil an Eiweisskörper gebunden ist. Die Eiweisskörper der Schilddrüse sind zuerst von Bubnow ${ }^{1}$ ) untersucht worden, welcher sie mit Wasser, Salzlösung und verdünnter Kalilauge extrahirte. Er erhielt auf diesem Wege drei verschiedene Körper, die er als Thyreoproteïde bezeichnete. Einer derselben zeigte die Eigenschaften eines Globulins.

Notkin ${ }^{2}$ ) beschrieb einen zusammengesetzten Eiweisskörper, welchen er durch eine nicht näher angegebene Behandlnng aus der Schilddrüse isolirte und Thyreoproteïd nannte. Nach seiner Ansicht ist das Thyreoproteïd ein Product des allgemeinen Stoffumsatzes und das Gift, welches sich nach der Schilddrüsenextirpation im Körper anhäuft und die Erscheinungen der thyreopriven Cachexie verursachen soll. Nach der -Auffassung von N otkin soll dieses Thyreoproteïd unter normalen Verhältnissen von dem eigentlichen Schilddrüsensecret, welches ein Enzym, das Thyreoidin, enthalte, zerstört resp. entgiftet werden. Dieses Enzym wäre also nach Notkin der specifisch wirksame Bestandtheil der Schilddrüse. An einer anderen Stelle (Referat in der Apothekerzeitung l. c.) heisst es : "Das Thyreoidin besteht aus mindestens zwei Eiweisskörpern, von denen der eine die Eigenschaften der Globuline besitzt, während der andere physiologisch wichtigere von enzymatischer Natur ist».

Unsere Untersuchungen haben uns $\mathrm{zu}$ ganz anderen Resultaten geführt, welche von den Ansichten Notkin's principiell verschieden sind.

Wir haben uns überzeugt, dass die wirksame Substanz der Schilddrüse mit einem Enzym oder ähnlichen Körpern ganz und gar Nichts zu thun hat, und dass es nur einen specifisch wirksamen Stoff in der Schilddrüse gibt, das Thyrojodin ${ }^{3}$ ),

1) Diese Zeitschr., Bd. 8, S. 1.

2) Wiener med. Wochenschr. 1895, Nr. 19 und 20, und ApothekerZeitung 1896, Nr. 13.

8) Damit soll nicht gesagt sein, dass alle anderen Substanzen, welche aus der Schilddrüse dargestellt werden können, physiologisch vollig indifferent sein müssen. 
welches theils frei, theils an Eiweisskörper gebunden in der Drüse sich befindet, und durch die Magensaftverdauung von den Eiweisskörpern - ebenso wie durch die Behandlung mit starken Säuren - abgespalten wird.

Durch zahlreiche Beobachtungen ist festgestellt worden: dass das Thyrojodin alle diejenigen Erscheinungen her vorzurufen vermag, welche bei der Schilddrüsentherapie bis jetzt als characteristische erkannt worden sind: Hierher gehören: 1. Die Wirkung auf Kröpfe. 2. Die Beeinflussung des Stoffwechsels bei Thieren ${ }^{1}$ ) und Menschen ${ }^{2}$ ). 3. Bei grösseren Dosen Vergiftungserscheinungen besonderer Art und last not least 4. die specifische Wirkung der Schilddrüsen bei Myxoed em. Letztere ist eine hier sehr selten vorkommende Erkrankung. Herr Prof. Leichtenstern in Köln hatte die grosse Freundlichkeit, mit dem Thyrojodin Beobachtungen bei Myxoedem anzustellen, wobei, wie er dem Einen von uns gütigst mittheilte, die Wirksamkeit des Thyrojodins sich als unzweifelhaft ergab.

Ueber die an Menschen und Thieren gemachten Beobachtungen wird der Eine von uns demnächst eingehend berichten.

Extrahirt man die zerkleinerten Schilddrüsen $3-4 \mathrm{mal}$ mit 0,75 procentiger Kochsalzlösung, so gelingt es, alle jodhaltigen Verbindungen in Lösung zu bringen. Der ungelöst bleibende Rückstand ist entweder ganz frei oder enthält nur noch Spuren von Jod. In letzterem Falle zeigt er in grösseren Mengen noch eine schwache Wirkung auf Kröpfe.

Verdünnt man den Salzwasserauszug mit dem 15 fachen Volumen Wasser, so trübt er sich und beim Einleiten von Kohlensäure fällt in feinen Flocken, welche bald zu fibrinartigen Gerinnseln sich vereinigen, die schon von Bubnow beobachtete Globulinsubstanz, aus, welche auch Notkin nachgewiesen hat.

1) Vergl. Roos, diese Zeitschr., Bd. 21, S. 19.

2) Treupel, Münchener med. Wochenschr. 1896, Nr. 6. 
Die Globulinsubstanz ist jodhaltig. Sie enthält den kleineren Theil des in der Drüse vorhandenen Thyrojodins. Beim Kochen mit Schwefelsäure wird aus derselben Thyrojodin abgeschieden. den. Gegen parenchymatöse Kröpfe ist dieses Globulin ungefähr ebenso wirksam wie das Thyrojodin selbst und wie die ganze Schilddrüse. Dieselbe Globulinsubstanz wird in etwas reichlicherer Menge durch Sättigen dès Salzwasserauszuges mit Magnesiumsulfatkrystallen abgeschieden.

Die von der Globulinsubstanz abfiltrirte Lösung gibt nach dem Ansäuern mit Essigsäure beim Kochen eine reichliche Coagulation eines Eiweisskörpers, welche gleichfalls jodhaltig ist und die Hauptmenge des Thyrojodins gebunden enthält. Bei der Verdauung dieser Albuminsubstanz mit Magensaft oder beim Kochen mit verdünnter Schwefelsäure $(1: 10)$ erhält man die grösste Ausbeute von dem in den Drüsen enthaltenen Thyrojodin. Der zweite Eiweisskörper zeigt im übrigen die Eigenschaften eines Albumins und ist bei Kröpfen wirksam.

Wird die von dem coagulirten Albumin abfiltrirte wässerige Lösung zur Trockne verdunstet, so erweist sich der Rückstand als jodfrei und unwirksam. Dasselbe Resultat ergibt sich, wenn man den Salzwasserauszug ohne vorherige Abscheidung des Globulins direct zur Coagulation bringt. Auch in diesem Falle ist das Filtrat jodfrei und unwirksam. Dabei wird also die Gesammtmenge des in der Drüse enthaltenen Thyrojodins mit den Eiweisskörpern abgeschieden. Die so erhaltenen Eiweissniederschläge können mit Vortheil für die weitere A bscheidung des Thyrojodins durch künstliche Verdauung oder durch Kochen mit Schwefelsäure verwerthet werden.

Durch Auskochen mit Alkohol wird dem coagulirten Eiweiss das darin enthaltene freie Thyrojodin entzogen, das vermöge seiner geringen Löslichkeit in Wasser gleichzeitig mit den Eiweisskörpern ausgefällt wird.

Aus unseren Beobachtungen geht somit hervor, dass das Thyrojodin in kleinerer Menge als solches in der Drüse sich findet; der weitaus grösste Theil derselben ist an einen Albuminstoff, welchen wir Thyrojodalbumin nennen, ein 
kleinerer Theil an eine Globulinsubstanz, das Thyrojodglobulin, gebunden.

\section{Ueber die Bestimmung des Jods in der Schilddrüsse.}

Für die therapeutische Verwerthung des Thyrojodins ist der Umstand, dass es sich um eine Jodverbindung handelt, von grösster Bedeutung. Denn dadurch wird eine constante Dosirung, die bei der Anwendung der Schilddrüse und ihrer Extracte ausgeschlossen war, ermöglicht.

Unsere Untersuchungen haben uns darüber belehrt, dass die Schilddrüsen der Hämmel (und anderer Thiere) nicht immer dieselbe Menge von Thyrojodin enthalten und dass sehr erhebliche Unterschiede bei Hämmeln überhaupt und besonders bei solchen, welche aus verschiedenen Gegenden stammen, in dieser Hinsicht sich zeigen.

Ungleiche Ausbeuten an Thyrojodin bei verschiedenen Darstellungen haben uns schon früher auf diesen Umstand aufmerksam gemacht, der erst durch die folgenden Untersuchungen in ein klares Licht gestellt wird.

Bei der Auswahl einer Methode für die directe Ermittelung des Jods in der Schilddrüse haben wir von gewichtsanalytischen Bestimmungen von vornherein abgesehen, weil es sich oft um Mengen, die nur Bruchtheile eines Milligramms betragen, in der zu untersuchenden oder zu Gebote stehenden Substanz handelte. Nach einigen Vorversuchen erkannten wir, dass das von Rabourdin ${ }^{1}$ ) empfohlene colorimetrische Verfahren sich am besten für unsere Zwecke eignete. Es besteht darin, dass das Jod nach Beseitigung der organischen Stoffe durch Schmelzen mit Alkali und Salpeter durch Ansäuern der wässerigen Lösung der Schmelze mit Schwefelsäure abgeschieden und mit einer bestimmten Menge von Chloroform ausgeschüttelt wird. Die Intensität der Färbung des Chloroforms entspricht innerhalb bestimmter Grenzen dem Jodgehalt, der durch Vergleichung mit der aus einer bekannten Menge von Jodkalium erzielten Färbung einer gleichen Menge

1) Lieb. Ann, 76, S. 375. 
von Chloroform ermittelt wird. Wir bedienten uns dabei gleich grosser Glascylinder, welche bei 20 Centimeter Höhe ungefähr $100 \mathrm{cbcm}$. Inhalt besassen.

Um die hierbei zu erzielende Genauigkeit zu prüten, wurden folgende Versuche angestellt:

1. Ein Gramm trockenes Fibrin wurde in einen Silbertiegel gebracht, $5 \mathrm{cbcm}$. einer Lösung von $0,1 \mathrm{gr}$. Jodkalium in 1 Liter, welche also 0,5 Milligramm Jodkalium enthielt, und 2 gr. Aetznatron hinzugefügt. Nachdem noch eine kleine Menge von Salpeter (ca. $1 / 2$ gr.) zugesetzt war, wurde der Tiegel vorsichtig erhitzt, bis Abscheidung von Kohle eintrat. Durch allmäligen weiteren Zusatz kleiner Mengen von Salpeter wird die Verbrennung beendigt. Die Schmelze wird in ca. 25 bis $30 \mathrm{cbcm}$. Wasser gelöst, die Lösung, wenn nöthig, filtrirt, abgekühlt, mit $10 \mathrm{cbcm}$. Chloroform versetzt, mit Schwefelsäure $(1: 5)$ angesäuert und gut durchgeschüttelt. Man giesst die Flüssigkeit nunmehr in einen der genannten Cylinder und ermittelt, wie viel von der obigen Jodkaliumlösung in einem zweiten Cylinder, welcher $10 \mathrm{cbcm}$. Chloroform und $50 \mathrm{cbcm}$. Wasser, das mit etwas Schwefelsäure und einigen Tropfen Natriumnitrit versetzt ist, hinzugefügt werden muss, um eine gleich starke Färbung des Chloroforms hervorzurufen.

Im vorliegenden Falle ergab sich eine gleich starke Färbung des Chloroforms in beiden Cylindern, nachdem bei der Vergleichsprobe 4,2 cbcm. der Jodkaliumlösung verbraucht waren. Es ergab sich also ein Verlust durch die Schmelzoperation von 0,08 Milligramm Jodkalium.

2. Ein Gramm trockenes Fibrin wurde nach Zusatz von 1 Milligramm Jodkalium, wie oben geschildert wurde, mit Aetznatron und Salpeter verascht. Die wiedergefundene Menge entsprach 0,9 Milligramm Jodkalium.

Danach hat man bei den hier in Betracht kommenden Mengen mit einem Verluste, welcher 0,08 bei 0,1 Milligramm Jodkalium (oder 0,06 bis 0,076 Milligramm Jod) entspricht, zu rechnen.

Die Erkennung der Farberiunterschiede ist am deutlichsten, wenn auf $10 \mathrm{cbcm}$. Chloroform nicht weniger als 0,2 Milli- 
gramm und nicht mehr als 1,5 Milligramm Jod kommen. Bei grösserem Gehalt an Jod sind die Färbungen so intensiv, dass man bei den von uns angewendeten Gefässen kleinere Unterschiede nicht mehr scharf erkennt. In solchem Falle sind Cylinder von geringerer Weite $\mathrm{zu}$ nehmen, oder $20 \mathrm{cbcm}$. Chloroform anzuwenden, oder man wiederholt den Versuch mit einer kleineren Menge der Substanz ( $1 /$, oder $1 / 4)$. Umgekehrt verfährt man, wenn die Menge des vorhandenen Jods wesentlich unter 0,2 Milligramm heruntergeht.

Bei einiger Uebung kann man es leicht erreichen, Unterschiede, welche $1 / 10$ Milligramm Jodkalium (= 0,076 Milligr. Jod) betragen, recht gut wahrzunehmen.

Die geschilderte Methode ist also, wie aus dem Vorstehenden sich ergibt, nicht überaus genau. Sie hat dafür den Vortheil, dass sie sehr schnell ausführbar ist und für die annähernde Ermittelung des Jodgehaltes der Schilddrüsen sich vortrefflich eignet. Bei sehr geringem Jodgehalt der zu prüfenden Substanz bemerkt man nicht selten, dass die Farbe der Chloroformlösung schnell abnimmt und verblasst; die Bestimmung muss daher ohne Unterbrechung zu Ende geführt werden. In solchen Fällen empfiehlt es sich, aus der Lösung der Schmelze, ohne vorher $\mathrm{zu}$ filtriren, nachdem man mit Salpetersäure angesăuert hat, das Jod als Jodsilber abzuscheiden, letzteres mit Zinkstaub und Schwefelsäure zu reduciren, und in der abfiltrirten Lösung die Jodbestimmung colorimetrisch in der oben angegebenen Weise auszuführen.

\section{Ueber den Jodgehalt der Hammelschilddrüsen.}

$\mathrm{Zu}$ diesen Versuchen benutzten wir Schilddrüsen, die $\mathrm{zu}$ verschiedenen Zeiten im hiesigen Schlachthause gesammelt worden sind (Tab. Nr. 1-3), ferner solche von Hammeln aus dem Wupperthal (Nr. 4-5) und solche, welche aus einem Schlachthause in Paris herrührten (Nr. 6 und 7). Die zuletzt genannten Proben der Hammelschilddrüsen sind grösseren Partien entnommen, welche wir den Farbenfabriken vorm. Fr. Bayer \& Go. in Elberfeld zu verdanken haben.

Zeitschrift für physiologische Chemie. XXI. 
Das Gewicht der durch Präpariren vom Fett befreiten Drüsen schwankte im Allgemeinen zwischen 3 und 5 gr. Man findet oft Drüsen, bei denen der eine Lappen erheblich kleiner als der andere ist.

100 Gewichtstheile frischer Drüsen ergaben bei $3 \mathrm{Be}-$ stimmungen von verschiedenen Drüsenpartien 28,2 bis 30,4 Gewichtstheile Trockensubstanz. Der Durchschnittswerth für letztere beträgt 29,5 Procent.

Zur Jodbestimmung diente gewöhnlich 1 gr. der bei $100^{\circ}$ getrockneten Drüsen; bei grösserem Jodgehalt wurde der Versuch mit 0,5 oder 0,2 gr. Substanz wiederholt. Alle Bestimmungen wurden doppelt ausgeführt, wobei nie grössere Unterschiede in den Resultaten sich zeigten, als etwa 0,07 Milligramm Jod entsprechen würde. Eine Correction für den beim Schmelzen zu erwartenden kleinen Verlust an Jod wurde nicht angebracht. Die Ergebnisse sind aus folgender Tabelle ersichtlich:

\begin{tabular}{|c|c|c|c|}
\hline \multirow[b]{2}{*}{ Nr. } & \multirow{2}{*}{$\begin{array}{c}\text { Herkunft } \\
\text { der } \\
\text { Schilddrüsen. }\end{array}$} & \multicolumn{2}{|c|}{ Jodgehalt in Milligramm } \\
\hline & & $\begin{array}{c}\text { von } 1 \mathrm{gr} . \\
\text { der trockenen } \\
\text { Drüse. }\end{array}$ & $\begin{array}{l}\text { von } 1 \text { gr. } \\
\text { der frischen } \\
\text { Drüse. }\end{array}$ \\
\hline 1. & Freiburg .... & 0,9 & 0,26 \\
\hline 2. & desgl. . . . & 1,0 & 0,29 \\
\hline 3. & desgl. . . . . . & 1,3 & 0,38 \\
\hline 4. & Elberfeld . . . . & 1,5 & 0,44 \\
\hline 5. & desgl. . & 5,3 & 1,56 \\
\hline 6. & Paris ... & 1,15 & 0,34 \\
\hline 7. & desgl. . . . & 1,2 & 0,35 \\
\hline
\end{tabular}

Aus diesen Bestimmungen geht hervor, dass der Jodgehalt der Schilddrüsen überhaupt und je nach ihrer Provenienz erheblichen Schwankungen unterliegen kann. Auch wenn man den bei $\mathrm{Nr} .5$ gefundenen Werth als einen ganz exceptionell hohen, der vielleicht nur sehr selten sich wieder findet, anzusehen hat, bleiben immer noch Schwankungen von 0,26 bis 0,44 übrig.

Bei der Vergleichung der Wirksamkeit des Thyrojodins mit derjenigen der frischen Drüsen wurden die hier gesam- 
melten Drüsen verwendet. Dabei ergab sich eine sehr gute Uebereinstimmung, wenn 0,25 bis 0,3 Milligramm Jod in Form von Thyrojodin an Stelle von je 1 gr. frischer Schilddrüsen verabreicht wurden.

Bei den überaus geringen Mengen, in welchen dás Thyrojodin dem Körper zugeführt werden muss, ist es geboten, dasselbe in einer Verreibung anzuwenden. Das von den Farbenfabriken vorm. Friedr. Bayer \& Co. uns gelieferte Thyrojodin enthält in $1 \mathrm{gr}$. der Milchzuckerverreibung 0,3 Milligramm Jod, wonach seine Wirkung derjenigen eines gleichen Gewichtes der frischen Drüsen, welche wie die hiesigen einen relativ niedrigen Jodgehalt besitzen, entsprechen würde.

Bei der Anwendung dieses Thyrojodins hat sich die bemerkenswerthe Erfahrung herausgestellt, dass dasselbe bei parenchymatösen Kröpfen seine Wirksamkeit schneller als die frischen Schilddrüsen oder die Tabletten entfaltet. Für die Erklärung dieser Erscheinung kommt wohl in Betracht, dass die Abspaltung des Thyrojodins aus den Eiweisskörpern der Schilddrüse durch die Verdauungsprocesse nur allmälig und vielleicht auch unvollständig erfolgt, und dass durch die Fäulnissprocesse des Darms ein Theil des Thyrojodalbumins in unwirksame Producte verwandelt werden kann.

Behandelt unan Thyrojodin in alkalischer Lösung mit Natriumamalgam, so wird Jodnatrium gebildet. Die Bedingungen für eine solche Zersetzung sind aber auch durch die Fäulnissprocesse gegeben, welche im Darm sich abspielen.

Das Thyrojodin selbst wird dagegen offenbar so schnell resorbirt, dass es den Fäulnissprocessen im Darm gar nicht ausgesetzt wird.

Freiburg i. B., den 2. März 1896.

Nachschrift. Während des Druckes dieser Mittheilung hat auch Herr Prof. Ewald mich benachrichtigt, dass er Myxoedem mit Thyrojodin erfolgreich behandelt habe. Für die Erlaubniss, dieses Resultat hier anführen zu dürfen, bin ich Herrn Prof. Ewald zu bestem Danke verpflichtet.

Baumann. 